57 BYSTANDER CARDIOPULMONARY RESUSCITATION AND LONG-TERM OUTCOMES IN OUT-OF-HOSPITAL CARDIAC ARREST ACCORDING TO LOCATION OF ARREST

${ }^{1}$ Kathrine B Sondergaard* ${ }^{*}$ 1,2Mads Wissenberg, ${ }^{3}$ Thomas Alexander Gerds, ${ }^{1}$ Shahzleen Rajan, ${ }^{1,2}$ Lena Karlsson, ${ }^{4,5}$ Kristian Kragholm, ${ }^{4}$ Marianne Pape, ${ }^{2}$ Freddy K Lippert, ${ }^{1,6,7}$ Gunnar H Gislason, ${ }^{1,2}$ Fredrik Folke, ${ }^{4,5,8}$ Christian Torp-Pedersen, ${ }^{4}$ Steen Moller Hansen. 'Department of Cardiology, Copenhagen University Hospital Gentofte, Denmark; ${ }^{2}$ Emergency Medical Services, Copenhagen, the Capital Region of Denmark; ${ }^{3}$ Department of Biostatistics, University of Copenhagen, Copenhagen; ${ }^{4}$ Unit of Epidemiology and Biostatistics, Aalborg University Hospital, Denmark; ${ }^{5}$ Department of Cardiology, Aalborg University Hospital, Denmark; ${ }^{6}$ National Institute of Public Health, University of Southern Denmark, Copenhagen, Denmark; ${ }^{7}$ The Danish Heart Foundation, Copenhagen, Denmark; ${ }^{8}$ Department of Health Science and Technology, Aalborg University, Denmark

10.1136/10.1136/bmjopen-2018-EMS.57

Aim Bystander cardiopulmonary resuscitation (CPR) has increased in several countries following nationwide initiatives to facilitate bystander resuscitative efforts in out-of-hospital cardiac arrest (OHCA). We examined the importance of public or residential location of arrest on temporal changes in bystander CPR and outcomes.

Method From the nationwide Danish Cardiac Arrest Registry, all OHCAs from 2001-2014 of presumed cardiac cause and between 18-100 years of age were identified. Arrests witnessed by emergency medical services personnel were excluded.

Results Of 25,505 OHCAs, 26.4\% $(n=6,738)$ and $73.6 \%$ $(\mathrm{n}=18,767)$ were in public and residential locations, respectively. Bystander CPR increased during 2001-2014 in both locations: from 36.4\% (95\% confidence interval [CI] 30.6\% to $42.6 \%$ ) to $83.1 \%$ (95\% CI: $80.0 \%$ to $85.8 \%$ ) in public $(\mathrm{p}<0.001)$ and from $16.0 \%$ (95\% CI: $13.2 \%$ to $19.3 \%)$ to $61.0 \%$ (95\% CI: $58.7 \%$ to $63.2 \%)$ in residential locations $(\mathrm{p}<0.001)$. Concurrently, 30 day survival increased in public from $6.4 \%$ (95\% CI: $4.0 \%$ to $10.0 \%)$ to $25.2 \%$ (95\% CI: $22.1 \%$ to $28.7 \%)(\mathrm{p}<0.001)$, and in residential from $2.9 \%$ (95\% CI: $1.8 \%$ to $4.5 \%)$ to $10.0 \%$ (95\% CI: $8.7 \%$ to $11.4 \%) \quad(\mathrm{p}<0.001)$. Among 2,28130 day survivors, one-year risk of anoxic brain damage/nursing home admission during 2001-2014 decreased from 18.8\% (95\% CI: $6.6 \%$ to $43.0 \%$ ) to $6.8 \%(95 \% \mathrm{CI}: 3.9 \%$ to $11.8 \%)$ in public $(\mathrm{p}<0.001)$, whereas the corresponding change was insignificant in residential locations from $11.8 \%$ (95\% CI: $3.3 \%$ to 34.3 ) to $17.6 \%$ (95\% CI: $12.7 \%$ to $23.9 \%)(\mathrm{p}=0.52)$.

Conclusion During 2001-2014, bystander CPR and 30 day survival more than doubled in both public and residential OHCA locations. A significant decrease in anoxic brain damage/nursing home admission was observed among 30 day survivors in public, but not among survivors from residential OHCAs.

Conflict of interest None

Funding Trygfonden Foundation KB Sondergaard.

\section{MIGHT AMBULANCE SERVICE ORGANISATIONAL CULTURE AFFECT AMBULANCE NON CONVEYANCE RATES?}

E Knowles*, L Bishop-Edwards, N Ahmed, A O'Cathain. SHARR, University of Sheffield, UK

\subsection{6/10.1136/bmjopen-2018-EMS.58}

Aim Ambulance services are experiencing increasing volumes of emergency calls. In response, they have increased the proportion of patients not conveyed to an emergency department (ED).
Alternatives to transporting patients to an ED include the provision of telephone advice only, treatment at the scene, or transport to another facility. Variation in the rates of different types of nonconveyance (NC), and in NC overall, exists between ambulance services in England. We explored variability in perceptions of the organisational culture within each individual ambulance service and show this in the context of NC rates.

Method We undertook 50 qualitative telephone interviews with ambulance service providers and commissioners in the 10 larger ambulance services in England, to identify factors that may explain variation in rates of ambulance NC. Data was analysed using a Framework model.

Results Amongst interviewees there was variation in the perceptions they held regarding the organisational culture of the ambulance service which they were employed by. Recent management restructuring within the ambulance service, perception of management support across the organisation, motivation of the organisation to undertake NC, and perceptions of support for staff undertaking NC varied across the ambulance services. There appeared to be a relationship between some of these factors and NC rates.

Conclusion Organisational culture is a complex issue but one which is potentially modifiable. Building a greater understanding of ambulance service organisational culture and how this relates to ambulance NC may help identify ways of improving service delivery across all ambulance services.

Conflict of interest None

Funding National Institute for Health Research (NIHR) Health Services and Delivery Research Programme.

\section{AMBULANCE OVER-CONVEYANCE TO THE EMERGENCY DEPARTMENT: A LARGE DATA ANALYSIS OF AMBULANCE JOURNEYS}

${ }^{1,2} \mathrm{~J}$ Miles ${ }^{*},{ }^{1} \mathrm{C} O \mathrm{O}^{\prime} \mathrm{Keeffe},{ }^{1} \mathrm{R}$ Jacques, ${ }^{1} \mathrm{~T}$ Stone, ${ }^{1} \mathrm{~S}$ Mason. ${ }^{1}$ University of Sheffield Centre for Urgent and Emergency Care Research; ${ }^{2}$ Yorkshire Ambulance Service

\subsection{6/10.1136/bmjopen-2018-EMS.59}

Aim Over-conveyance by the ambulance service is a compounding factor of emergency department (ED) crowding. Previous solutions have focused on specific patient groups which have a limited impact when compared to the whole urgent and emergency care system. This study aims to analyse nonurgent conveyances by the ambulance service that could be suitable for discharge on-scene.

Results We analysed a dataset of 1,312,539 patient episodes which linked all pre-hospital emergency and urgent calls to subsequent ED attendance in 2014. The study was set in a large region in England (total population 5.3 million). As well as proportion of avoidable conveyances we also examined the association with patient age, time of arrival, re-attendance and initial triage code from ambulance dispatch.

Results There were 404348 (30.8\%) patients transported to ED by ambulance and of these $66220(16.4 \%)$ were considered potentially avoidable. There were significantly increased odds of a non-urgent conveyance out of hours (OR 1.44, 95\% CI: 1.41 to 1.46). Patients aged $16-34$ had the largest proportion of avoidable conveyances with 24500 (37\%). There were 13625 (21\%) episodes that were received from another healthcare professional or urgent telephone number. When analysing ED diagnosis, the highest proportion were attending with minor injury and illness, and alcohol intoxication. 